\title{
Atypical (Mild) Forms of Dihydropteridine Reductase Deficiency: Neurochemical Evaluation and Mutation Detection
}

\author{
NENAD BLAU, CLAUS W. HEIZMANN, WOLFGANG SPERL, GEORG C. KORENKE, \\ GEORG F. HOFFMANN, PETER M. SMOOKER, AND RICHARD G. H. COTTON

\begin{abstract}
University Children's IIospital, Division of Clinical Chemistry, Zurich, Switzerland [N.B., C.W.H.]; Department of Pediatrics, University of Innsbruck, Austria [W.S.]; Department of Pediatrics and Child Neurology, University of Gottingen, Germany [G.C.K.]; Department of Pediatrics, University of Heidelberg, Germany [G.F.H.]; and
\end{abstract} \\ Olive Miller Laboratory. The Murdoch Institute, Roval Children's Hospital, Melbourne, Australia
} [P.M.S., R.G.H.C.]

\begin{abstract}
We investigated two patients with an atypical (mild) form of dihydropteridine reductase (DHPR) deficiency. Both responded to the loading test with tetrahydrobiopterin; their plasma phenylalanine levels were lowered from $278 \mu \mathrm{mol} / \mathrm{L}$ to 85 and $48 \mu \mathrm{mol} / \mathrm{L}$ and from $460 \mu \mathrm{mol} / \mathrm{L}$ to 97 and $36 \mu \mathrm{mol} / \mathrm{L}$ after 4 and $8 \mathrm{~h}$, respectively. In one of the patients, a combined loading test with phenylalanine followed by tetrahydrobiopterin was also carried out and showed a profile typical for DHPR deficiency. The phenylalanine hydroxylation rate was calculated to be 43 and $87 \%, 4$ and $8 \mathrm{~h}$ after cofactor administration, respectively. Diagnosis was confirmed by the absence of DHPR activity in the patient's erythrocytes. In cultured fibroblasts, residual activity of 4 and $10 \%$, respectively, was found. Excretion of urinary pterins was essentially normal, and the biopterin to neopterin ratio in cerebrospinal fluid was increased. Although in both patients cerebrospinal fluid homovanillic acid was found to be normal, and 5-hydroxyindoleacetic acid was substantially reduced, there was no sign of neurologic alterations until the age of $2 \mathrm{y}$. However, one of the patients recently developed deceleration of head growth, whereas psychomotor development continued to be normal for age. Using the chemical cleavage method on the amplified cDNA, mismatches of $T$ to $G$ at nucleotide 659 and of $G$ to $A$ at nucleotide 475 , respectively, were identified. These results also demonstrate that screening for tetrahydrobiopterin deficiency by urinary pterin analysis alone can miss some newborns with mild DHPR deficiency and that all children with tetrahydrobiopterin defects need full neurochemical evaluation together with analysis of the enzyme activity. (Pediatr Res 32: 726-730, 1992)
\end{abstract}

\section{Abbreviations}

DHPR, dihydropteridine reductase

$\mathrm{BH}_{4}$, tetrahydrobiopterin

PKU, phenylketonuria

PCR, polymerase chain reaction

CSF, cerebrospinal fluid

5HIAA, 5-hydroxyindoleacetic acid

HVA, homovanillic acid

Received March 3, 1992; accepted July 2, 1992

Correspondence: Dr. Nenad Blau, University Children's Hospital, Steinwiesstrasse 75, CH-8032 Zurich, Switzerland.

Supported by the Swiss National Science Foundation, Grant 31-28797.90 and in part by the Helmut Horten Research Foundation.
Hyperphenylalaninemia (1) comprises a group of autosomalrecessively inherited disorders caused either by a defect of the phenylalanine-4-hydroxylase (classic PKU) or by deficiency of the cofactor $\mathrm{BH}_{4}$. Two defects are known to occur in the biosynthesis of $\mathrm{BH}_{4}$, i.e. GTP cyclohydrolase I deficiency and 6-pyruvoyl tetrahydropterin synthase deficiency. The latter is known to be the most common form of $\mathrm{BH}_{4}$-dependent hyperphenylalaninemia (2). In DHPR deficiency, the second most common form of $\mathrm{BH}_{4}$ deficiency, quinonoid-dihydrobiopterin formed during the hydroxylation of phenylalanine to tyrosine accumulates, and the amount of $\mathrm{BH}_{4}$ synthesized by the de novo pathway is insufficient for the normal function of hepatic phenylalanine4-hydroxylase (3). Because $\mathrm{BH}_{4}$ is also the essential cofactor of tyrosine-3-hydroxylase and tryptophan-5-hydroxylase, these patients are generally characterized by depletion of the monoamine neurotransmitters dopamine, norepinephrine, and serotonin $(4,5)$.

In contrast to patients with classic PKU, which can be successfully treated with the low-phenylalanine diet, patients with DHPR deficiency need different treatment. They do not respond to the phenylalanine-restricted diet and, if not diagnosed early and treated properly, are subject to severe myoclonic epilepsy and mental retardation, which often lead to early death (6). Furthermore, several reports demonstrate partial and mild cases of DHPR deficiency $(7,8)$; however, no neurochemical evaluations were performed in these patients and there are no data on the type of mutation at the molecular level.

In this article, we describe the clinical and biochemical data of two patients with atypical (mild) forms of DHPR deficiency, one treated with $\mathrm{BH}_{4}$ supplementation and the other not treated. Data presented clearly demonstrate that: 1) measurement of DHPR activity in erythrocytes is essential for detection of DHPR deficient patients; 2) despite favorable clinical presentation, these patients show a discrepancy between serotonin and dopamine turnover; and 3) mRNA analysis performed on the patients' cultured fibroblasts revealed two different mutations despite identical clinical and biochemical presentation.

\section{MATERIALS AND METHODS}

Measurement of pterins in urine and CSF was performed after oxidation with manganese dioxide using reverse-phase HPLC with fluorescence detection (9). For the determination of tetrahydrobiopterin, samples were pretreated by differential oxidation (10). Neurotransmitter metabolites were measured by ion-pair HPLC (11). Amino acid analysis was performed by ion-exchange chromatography using Biotronik LC 5001.

The detection of enzyme mutations was performed by a chem- 
ical cleavage reaction of mismatches in heteroduplexes formed between normal and patients' RNA (12). After mRNA isolation from the patients' fibroblasts and cDNA synthesis, the PCR was used to amplify the coding region of the cDNA. Oligonucleotides used for the amplification were complementary to the cDNA from nucleotides 34 to 45 and 784 to 803 . The former also contained a 10-nucleotide tail with a $B a m H I$ restriction site. Hence, all except the first seven of the 244 amino acids were screened. The mutant PCR product was then screened for mismatches with the wild type cDNA (13).

DHPR activity in erythrocytes was determined by a spectrophotometric measurement of the quinonoid-6-methyldihydropterin-dependent oxidation of ferricytochrome $C$ (14). Cultured fibroblasts were assayed for DHPR activity (15). Additionally, both assay procedures were adapted using $\mathrm{BH}_{4}$ instead of 6methyltetrahydropterin.

$(6 \mathrm{R}, \mathrm{S})-\mathrm{BH}_{4}$ and 6-methyltetrahydropterin were purchased from Dr. B. Schircks Laboratories (Jona, Switzerland).

\section{CASE REPORTS}

Patient 1. M.LJ. is the third child of healthy, unrelated parents of Yugoslav origin. Family history, pregnancy, and birth (at term) were uneventful; birth weight was $2930 \mathrm{~g}$; length, $48 \mathrm{~cm}$; and head circumference, $34 \mathrm{~cm}$. The Apgar scores were 9, 10, and 10 at 1,5 , and $10 \mathrm{~min}$, respectively. In the postnatal period, hyperbilirubinemia $(306 \mu \mathrm{mol} / \mathrm{L})$, due to A0 incompatibility, was noted and treated with phototherapy. Hyperphenylalaninemia was detected by the Guthrie test in the course of the national screening program. It took $6 \mathrm{wk}$ to get a serum for quantitative amino acid analysis due to noncompliance of the parents. At the age of $2 \mathrm{mo}$, plasma phenylalanine concentration was $284 \mu \mathrm{mol} /$ L. Until the age of $21 \mathrm{mo}$, plasma phenylalanine ranged from 73 to $399 \mu \mathrm{mol} / \mathrm{L}$ while the patient was on a free diet. So far, the child shows a normal psychomotor development on a normal diet without any phenylalanine restriction and without substitution with $\mathrm{BH}_{4}$ or neurotransmitter precursors, growing along the 25 th percentile for all body measurements. At the age of $18 \mathrm{mo}$ magnetic resonance imaging of CNS revealed no abnormalities.

Patient 2. G.A. is the first child of Turkish parents, who are first cousins. Family history, pregnancy, and birth (weight, 3200 $\mathrm{g}$; length, $50 \mathrm{~cm}$; head circumference, $34 \mathrm{~cm}$, and Apgar scores, 9,10 , and 10 at 1,5 , and $10 \mathrm{~min}$, respectively) were uneventful. Hyperphenylalaninemia was detected by the neonatal screening program. At the age of $2 \mathrm{wk}$, the plasma phenylalanine concentration was $500 \mu \mathrm{mol} / \mathrm{L}$. The CSF concentration of the serotonin metabolite was substantially reduced, and cofactor replacement therapy was started with $10 \mathrm{mg} \mathrm{BH} / 4 / \mathrm{kg}$ body weight, given in a single dose. Tetrahydrofolate concentrations in serum measured at the age of $1,2,3$, and 9 mo were in the normal range (between 10.3 and $22.4 \mathrm{ng} / \mathrm{mL}$ ). There had been no restriction of phenylalanine in the diet. No abnormalities were found in cranial magnetic resonance imaging scans performed at the age of 1 and $2 \mathrm{y}$. Examination of auditory-, visual-, and sensory-evoked potentials performed every 6 mo was always normal. The psychomotor development up to the age of 30 mo was completely normal, but during the last year a deceleration of the head growth was observed. At the age of $30 \mathrm{mo}$, the patient was microcephalic with a head circumference $0.5 \mathrm{~cm}$ below the $3 \mathrm{rd}$ percentile. Body weight was between the 3rd and 10th percentile; length was between the 10 th and 25 th percentiles. Because of the reduction of serotonin biosynthesis in CSF and because of the developing microcephaly, additional therapy with 5-hydroxytryptophan $(2.5$ $\mathrm{mg} / \mathrm{kg}$ body weight/d) and folinic acid $(15 \mathrm{mg} / \mathrm{d})$ was started.

\section{RESULTS}

$\mathrm{BH}_{4}$ loading test. In patient M.LJ., both oral administration of $\mathrm{BH}_{4}(7.5 \mathrm{mg} / \mathrm{kg}$ body weight $)$ as well as combined oral loading with phenylalanine ( $100 \mathrm{mg} / \mathrm{kg}$ body weight) and, $3 \mathrm{~h}$ later, with
$\mathrm{BH}_{4}(20 \mathrm{mg} / \mathrm{kg}$ body weight $)$ showed a decrease of plasma phenylalanine after 4 and $8 \mathrm{~h}$ (Table 1 ). The hydroxylation rate of the administered phenylalanine after loading with $\mathrm{BH}_{4}$, calculated as the amount of phenylalanine converted to tyrosine, was found to be $43 \%(4 \mathrm{~h})$ and $87 \%(8 \mathrm{~h})$. In patient G.A., an oral loading test with $20 \mathrm{mg} \mathrm{BH}_{4} / \mathrm{kg}$ body weight showed similar normalization of plasma phenylalanine.

Pterins in urine and CSF. Urinary total neopterin and total biopterin were slightly increased in both patients. The pterin pattern was similar to that found in patients with classic PKU (Table 2). Percentage of biopterin (of the sum of neopterin and biopterin) was between 34 and $62 \%$ in the urine of patient M.LJ. and between 31 and $82 \%$ in the urine of patient G.A. However, patient G.A. had been on $\mathrm{BH}_{4}$ monotherapy $(10 \mathrm{mg} / \mathrm{kg}$ body weight/d) since the age of $3 \mathrm{mo}$. In the second patient, neopterin excretion decreased with age, whereas biopterin excretion increased. A percentage of biopterin in urine greater than 80 usually is characteristic of DHPR deficiency. The percentage of $\mathrm{BH}_{4}$ (of the sum of $\mathrm{BH}_{4}$, dihydrobiopterin, and biopterin) in the urine of patient M.LJ. was $7 \%$, and in patient G.A., $17 \%$, both markedly below the normal range of $\sim 60 \%$.

In the CSF, total neopterin was normal and total biopterin increased (Table 2), a pattern typical for DHPR deficiency. High neopterin in the CSF of patient G.A. at the age of 1 mo was probably due to a viral infection.

Neurotransmitter metabolites in CSF. The most interesting finding is that 5HIAA was markedly decreased, whereas HVA was in the normal range in both patients (Table 2). With increased age, values remained in the same range, and there was no correlation with either the changes in neopterin and biopterin content in the CSF or the plasma phenylalanine and tyrosine concentrations. These findings indicate diminished serotonin and normal catecholamine biosynthesis in the CNS, an unusual finding for patients with $\mathrm{BH}_{4}$ deficiency. Trial of cofactor replacement therapy $(10 \mathrm{mg} / \mathrm{kg}$ body weight $/ \mathrm{d})$ over $7 \mathrm{~d}$ in the patient G.A. resulted not only in a favorable control of hyperphenylalaninemia, but also in a slightly increased production of serotonin in the CSF, as was indicated by the CSF levels of 5HIAA (Table 2). Surprisingly, interruption of $\mathrm{BH}_{4}$ treatment for $4 \mathrm{~d}$ resulted in neither a substantial increase of plasma and CSF phenylalanine nor a drop of CSF neurotransmitter metabolites (data not shown). After 8 wk without any medication, 5HIAA in CSF was further reduced and HVA lowered to borderline. Despite excellent psychomotor development of the patient, treatment with $\mathrm{BH}_{4}$ was reintroduced at the age of $6 \mathrm{mo}$, resulting in a good control of the plasma phenylalanine. HVA completely normalized, whereas 5HIAA remained below the control value.

DHPR activity in erythrocytes and skin fibroblasts. Diagnosis of DHPR deficiency was confirmed by the measurement of

Table 1. Plasma phenylalanine and tyrosine after $\mathrm{BH}_{4}$ loads performed with and without oral administration of phenylalanine (Phe) in two children with atypical form of DHPR deficiency

\begin{tabular}{lrrr}
\hline & & \multicolumn{2}{c}{ Plasma $(\mu \mathrm{mol} / \mathrm{L})$} \\
\cline { 3 - 4 } & Time $(\mathrm{h})$ & Phe & Tyrosine \\
\hline Patient M.LJ. & -3 & 288 & 73 \\
Phe load $(100 \mathrm{mg} / \mathrm{kg})$ & 0 & 635 & 60 \\
BH $_{4}$ load $(20 \mathrm{mg} / \mathrm{kg})$ & 4 & 363 & 93 \\
& 8 & 85 & 82 \\
BH $_{4}$ load $(7.5 \mathrm{mg} / \mathrm{kg})$ & 0 & 278 & 105 \\
& 4 & 97 & 99 \\
Patient G.A. & 8 & 36 & 105 \\
BH & & & \\
& 0 & 460 & 88 \\
& 4 & 97 & 148 \\
& 8 & 36 & 93 \\
\hline
\end{tabular}


Table 2. Urinary and CSF pterins, CSF neurotransmitter metabolites, and plasma amino acids in two patients with atypical form of DHPR deficiency

\begin{tabular}{|c|c|c|c|c|c|c|c|c|c|}
\hline \multirow[b]{2}{*}{ Age (mo) } & \multicolumn{2}{|c|}{$\begin{array}{l}\text { Urine ( } \mathrm{mmol} / \mathrm{mol} \\
\text { creatinine) }\end{array}$} & \multicolumn{4}{|c|}{$\mathrm{CSF}(\mathrm{nmol} / \mathrm{L})$} & \multicolumn{2}{|c|}{ Plasma $(\mu \mathrm{mol} / \mathrm{L})$} & \multirow{2}{*}{$\begin{array}{c}\text { Therapy } \\
\mathrm{BH}_{4}(\mathrm{mg} / \\
\mathrm{kg} / \mathrm{d})\end{array}$} \\
\hline & Neopterin & Biopterin & Neopterin & Biopterin & 5HIAA & HVA & Phenylalanine & Tyrosine & \\
\hline \multicolumn{10}{|l|}{ Patient M.LJ. } \\
\hline 2 & 10.2 & 5.2 & 13 & 57 & 66 & 464 & 288 & 73 & \\
\hline 7 & 6.1 & 3.6 & 20 & 36 & 21 & 278 & 115 & 61 & \\
\hline 10 & 5.1 & 4.7 & 25 & 51 & 21 & 307 & 103 & 55 & \\
\hline 14 & 3.1 & 4.8 & 19 & 52 & 22 & 381 & 211 & 95 & \\
\hline \multicolumn{10}{|l|}{ Patient G.A. } \\
\hline 1 & 15.7 & 7.8 & 44 & 37 & 33 & 390 & 793 & 6 & \\
\hline 3 & 5.5 & 6 & 17 & 59 & 59 & 571 & 194 & 99 & 10 \\
\hline 4 & 4.5 & 7.1 & 14 & 44 & 34 & 377 & 133 & 66 & \\
\hline 13 & 1.7 & 7.7 & 12 & 24 & 38 & 440 & 36 & 26 & 10 \\
\hline 18 & 1.5 & 6.1 & 10 & 42 & 33 & 507 & 36 & 138 & 10 \\
\hline 24 & 1.1 & 3.1 & 8 & 26 & 30 & 477 & 54 & 50 & 10 \\
\hline Controls & $1.1-4.1$ & $0.5-3.1$ & $9-20$ & $10-30$ & $70-400$ & $300-1000$ & $<70$ & $<120$ & \\
\hline
\end{tabular}

enzyme activity in the patients' erythrocytes and cultured skin fibroblasts (Table 3 ). In the erythrocytes of both patients, no DHPR activity was detectable if either the synthetic (6-methyltetrahydropterin) or the natural $\left(\mathrm{BH}_{4}\right)$ cofactor was used in the assay. Both parents were diagnosed to be heterozygous for DHPR deficiency, showing a reduced enzyme activity of $\sim 50 \%$ of controls. In the cultured skin fibroblasts of the patients M.LJ. and G.A., a severely reduced DHPR activity of 4 and $10 \%$, respectively, was found.

Screening for nucleotide substitution. cDNA screening of the patients with DHPR deficiency was performed on PCR products that encompassed the coding region of the cDNA. In the patient G.A., a mismatch, reacting with osmium tetroxide at base 659 , corresponded to the conversion of $\mathrm{T}$ to $\mathrm{G}$ (Fig. 1), changing amino acid no. 212, phenylalanine (TTC), to cystine (TGC). In the patient M.LJ., a mismatch reacting with hydroxylamine at base 475 , corresponded to the conversion of $\mathrm{G}$ to A (Fig. 2), changing amino acid no. 151, glycine $(\mathrm{GGC})$, to serine $(\mathrm{G} A \mathrm{C})$. Both of the mutations are homozygous.

\section{DISCUSSION}

Two patients with DHPR deficiency described here demonstrate that every newborn with even slightly elevated plasma phenylalanine should be screened for $\mathrm{BH}_{4}$ deficiency. The first patients with $\mathrm{BH}_{4}$ deficiency were already identified in 1969 (16). Siblings with mild hyperphenylalaninemia, at that time described as "a genetic variant of phenylketonuria," were later characterized as DHPR deficient (17). Another child with severe neurologic symptoms, despite treatment with a low-phenylalanine diet,

Table 3. DHPR activity in erythrocytes and skin fibroblasts of two patients with atypical form of DHPR deficiency and their parents

\begin{tabular}{lcc}
\hline & $\begin{array}{c}\text { Erythrocytes } \\
(\mathrm{mU} / \mathrm{mg} \mathrm{Hb})^{*}\end{array}$ & $\begin{array}{c}\text { Fibroblasts } \\
(\mathrm{mU} / \mathrm{mg} \text { protein }) \dagger\end{array}$ \\
\hline Patient G.A. & $<0.5$ & 5 \\
Father & 1.4 & $\mathrm{ND} \ddagger$ \\
Mother & 1.5 & $\mathrm{ND}$ \\
Patient M.LJ. & $<0.5$ & 2 \\
Father & 1.2 & $\mathrm{ND}$ \\
Mother & 1.1 & $\mathrm{ND}$ \\
Controls & $2-5$ & $22-49$ \\
& $(n=29)$ & $(n=9)$ \\
\hline
\end{tabular}

* nmol cytochrome $c$ reduced $/ \mathrm{min} / \mathrm{mg}$ hemoglobin.

$\uparrow$ nmol NADH oxidized $/ \mathrm{min} / \mathrm{mg}$ protein.

$\ddagger N D$, not determined.

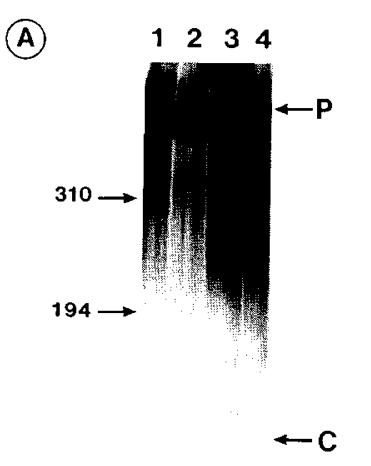

(B)

$118 \rightarrow$

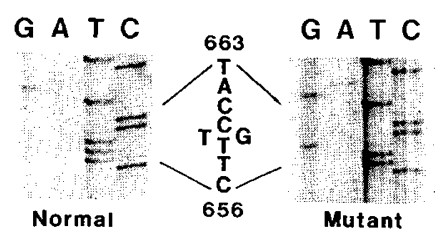

Fig. 1. Identification of a mutation in patient G.A. Panel $A$ shows an autoradiogram of the chemical cleavage method gel. Lanes 1 and 2 are normal controls; lanes 3 and 4 are from the patient. Lanes 1 and 3 are the result of hydroxylamine reaction; lanes 2 and 4 are the result of reaction with osmium tetroxide. The probe band is marked as $P$, and cleavage product can be seen in lane $4(C)$. The numbers on the lefthand side of the gel refer to molecular weight markers, in bp. Panel $B$ shows the nucleotide sequence around the estimated point of the mismatch. In the patient, the cDNA has a $\mathrm{T}$ to $\mathrm{G}$ substitution at nucleotide 659.
(A)

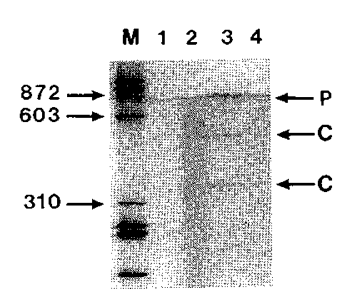

(B)

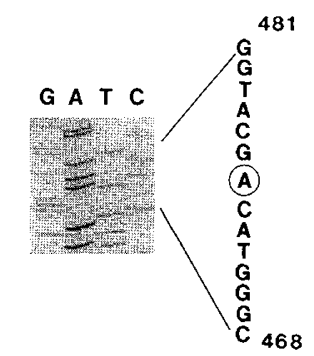

Fig. 2. Identification of a mutation in patient M.LJ. Panel $A$ shows an autoradiogram of a chemical cleavage method gel (abbreviations and labels are the same as in Figure 1A). A cleavage is evident in lane 3. In this case, both cleavage products are visible. Panel $B$ shows the nucleotide sequence around the estimated point of the mismatch. Only the sequence from the patient is shown. There is a $\mathrm{G}$ to A substitution at nucleotide 457 (circled). 
was found to have normal phenylalanine-4-hydroxylase but no DHPR activity in the liver (3). Other patients with DHPR deficiency have been described later (18-20), and, in one family, two subjects were found to have no DHPR activity in blood but were clinically normal (21). Unfortunately, this family refused further investigations aimed at defining the reason for this finding.

The $\mathrm{BH}_{4}$ loading test in patients with DHPR deficiency is not as effective as in patients with 6-pyruvoyl tetrahydropterin synthase deficiency. Because some of the DHPR-deficient patients did not respond to the dose of $7.5 \mathrm{mg} \mathrm{BH}_{4} / \mathrm{kg}$ body weight (2.2), loading with $20 \mathrm{mg} \mathrm{BH}_{4} / \mathrm{kg}$ body weight was introduced (23). However, one patient, described as a nonresponder even to this high dose of $\mathrm{BH}_{4}$, died despite an early diagnosis and treatment and may represent a "malignant" form of DHPR deficiency (24). There is a remote possibility that this patient may have had both phenylalanine hydroxylase and DHPR deficiency, which could also explain the lack of a response to the $\mathrm{BH}_{4}$ loading test. The differences in the response to oral loading with $\mathrm{BH}_{4}$ may be due to the gene dosage effect or more likely to the heterogeneity of the molecular defect as demonstrated previously $(22,25)$. In our patients, in both loading tests $(7.5 \mathrm{mg} / \mathrm{kg}$ body weight in patient M.LJ. and $20 \mathrm{mg} / \mathrm{kg}$ body weight in patient G.A.) plasma phenylalanine normalized $8 \mathrm{~h}$ after oral administration. The combined phenylalanine- $\mathrm{BH}_{4}$ loading test performed in patient M.LJ. at initially normal plasma levels showed hydroxylation rates typical for DHPR deficiency.

Distribution of CSF 5HIAA (a metabolite of serotonin) and HVA (a metabolite of dopamine) in patients with DHPR deficiency is similar to that observed in 6-pyruvoyl tetrahydropterin synthase deficiency. However, the decrease of 5HIAA seems to be more dramatic than that of HVA (6). Absence of DHPR activity in the erythrocytes of our patients confirmed the diagnosis, and we expected to find the abnormal profile of the neurotransmitter metabolites in the CSF. This expectation was only partially confirmed by very low levels of 5HIAA but normal HVA in the CSF of both children. Despite a low serotonin biosynthesis, a favorable clinical course, good psychomotoric development, and high phenylalanine tolerance were found in our patients. Furthermore, mild hyperphenylalaninemia and normal CSF phenylalanine levels (data not shown) in both patients exclude the competitive inhibition of tryptophan-5hydroxylase by phenylalanine. Because there were no signs of neurologic abnormalities, both children were kept on a normal diet. A trial with $\mathrm{BH}_{4}(10 \mathrm{mg} / \mathrm{kg}$ body weight/d) in patient $\mathrm{G} . \mathrm{A}$. resulted, as expected, in complete normalization of plasma phenylalanine levels. Surprisingly, production of serotonin increased toward normal, and HVA increased substantially. Because the penetration of $\mathrm{BH}_{4}$ into the brain is known to be poor, cofactor replacement therapy in DHPR deficiency is usually inefficient. Furthermore, in one of our patients reintroduction of $\mathrm{BH}_{4}$ therapy normalized only catecholamine production, whereas serotonin biosynthesis remained low. A partial DHPR deficiency associated with mental retardation was documented by abnormally low levels of 5HIAA in the CSF from patients with neurologic and mental disorders (7). The residual activity of DHPR in erythrocytes purified by affinity chromatography was found in a patient with only mild mental retardation without neurotransmitter therapy (8), suggesting that in mild forms of DHPR deficiency neurologic symptoms may occur at a later stage. Therefore, therapy has been started in one patient and will be started in the second despite normal development so far.

Recently, patient G.A. showed deceleration of head growth and, despite the good psychomotoric development and normal blood folate concentrations, we intend to introduce therapy with the neurotransmitter precursor 5-hydroxytryptophan and folinic acid. Folinic acid supplementation was shown to improve neurologic symptoms in some patients with DHPR deficiency (26), with prevention of intracranial calcification $(19,20,27)$. Because there is strong support for the belief that DHPR plays a role in maintaining tetrahydrofolate levels in the brain (28), folinic acid was recommended to be a part of the treatment in DHPRdeficient patients.

Clinical and neurochemical evaluation in our patients strongly supported the hypothesis that such atypical (mild) forms of DHPR deficiency may be due to the same type of mutation. While screening the CDNA of the DHPR-deficient patients, different types of mutations such as neutral mutation (polymorphism) of the leucine codon to another leucine codon and insertion of an extra codon for threonine were detected (13). In patient G.A., we found a mutation of a phenylalanine codon (T TC) to cystine codon (T $G \mathrm{C}$ ), and in patient M.LJ., a mutation of a glycine codon $(\mathrm{G} G \mathrm{C})$ to serine codon $(\mathrm{G} A \mathrm{C})$. These two mutations occur 61 amino acids apart in a protein region not responsible for the NADH binding. However, there is no direct evidence that this may be the $\mathrm{BH}_{4}$ binding domain. Therefore, it seems difficult to predict the severity of the disease on the basis of the type of mutation, at least in patients with DHPR deficiency.

The study of our patients with atypical DHPR deficiency demonstrates the importance of exact neurochemical evaluation of all children with $\mathrm{BH}_{4}$ deficiency. This includes the measurement of not only pterins in urine but also neurotransmitter metabolites in CSF. Furthermore, these patients should be controlled periodically during the first years of life regardless of their clinical status.

Acknowledgments. The authors thank Lucja Kierat, Ana Matasovic, Sönke Holm, and Gary Pfeifer for skillful technical assistance.

\section{REFERENCES}

1. Scriver CR, Kaufman S, Woo SLC 1990 The hyperphenylalaninemias. In Scriver CR, Beaudet AL, Sly WS, Valle D (eds) The Metabolic Basis of Inherited Disease, 6th Ed, Vol I. McGraw-Hill, New York, pp 495-546

2. Blau N 1988 Inborn errors of pterin metabolism. Annu Rev Nutr 8:185-209

3. Kaufman S, Holtzman NA, Milstien S, Butler IJ, Krumholz A 1975 Phenylketonuria due to a deficiency of dihydropteridine reductase. N Engl J Med 293:785-790

4. Koslow SH, Butler IJ 1977 Biogenic amine synthesis defect in dihydropteridine reductase deficiency. Science 198:522-523

5. Ponzone A, Guardamagna O, Ferraris S, Biasetti S, Bracco G, Niederwieser A 1987 Neurotransmitter therapy and diet in malignant phenylketonuria. Eur J Pediatr 146:93-94

6. Dhondt JL 1991 Register of tetrahydrobiopterin deficiency. Bagnolet: Milupa, pp 7-11

7. Sahota A, Leeming RJ, Blair JA, Armstrong RA, Green A, Cohen BE 1986 Partial dihydropteridine reductase deficiency and mental retardation. J Inherited Metab Dis 9(suppl 1):247-249

8. Nakabayashi H, Owada M, Kitagawa T 1984 A mild case of dihydropteridine reductase deficiency with residual activity in erythrocytes. J Inherited Metab Dis 7:135-136

9. Niederwieser A, Staudenmann W, Wetzel E 1984 High-performance liquid chromatography with column switching for the analysis of biogenic amine metabolites and pterins. J Chromatogr 290:237-246

10. Fukushima T, Nixon JC 1980 Analysis of reduced forms of biopterin in biological tissues and fluids. Anal Biochem 102:176-188

11. Blau N, Niederwieser A, Curtius HCh, Kierat L, Leimbacher W, Matasovic A, Binkert F, Lehmann H, Leupold D, Guardamagna O, Ponzone A, Schmid H, Coskun T, Ozalp I, Giugliani R, Biasucci G, Giovannini M 1989 Prenatal diagnosis of atypical phenylketonuria. J Inherited Metab Dis 12(suppl 2):295-298

12. Cotton RGH, Rodrigues NR, Campbell RD 1988 Reactivity of cytosine and thymine in single-base-pair mismatches with hydroxylamine and osmium tetroxide and its application to the study of mutations. Proc Natl Acad Sci USA 85:4397-4401

13. Howells DW, Forrest SM, Dahl HHM, Cotton RGH 1990 Insertion of an extra codon for threonine is a cause of dihydropteridine reductase deficiency. Am J Hum Genet 47:279-285

14. Arai N, Narisawa K, Hayakawa H, Tada K 1982 Hyperphenylalaninemia due to dihydropteridine reductase deficiency: diagnosis by enzyme assay on dried blood spots. Pediatrics 70:426-430

15. Firgaira FA, Cotton RGH, Danks DM 1979 Human dihydropteridine reductase. A method for the measurement of activity in cultured cells and its application to malignant hyperphenylalaninemia. Clin Chim Acta 95:47-59

16. Tada K, Yoshida T, Mochizuki K, Konno T, Nakagawa H, Yokoyama Y, Takada G, Arakawa T 1969 Two siblings of hyperphenylalaninemia: suggestion to a genetic variant of phenylketonuria. Tohoku J Exp Med 100:249253 
17. Tada K, Narisawa K, Arai N, Ogasawara Y, Ishizawa S 1980 A sibling case of hyperphenylalaninemia due to a deficiency of dihydropteridine reductase: biochemical and pathological findings. Tohoku J Exp Med 132:123-131

18. Danks DM, Schlesinger P, Firgaira F, Cotton RGH, Watson BM, Rembold $H$, Hennings G 1979 Malignant hyperphenylalaninemia: clinical features, biochemical findings, and experience with administration of biopterins. Pediatr Res 13:1150-1155

19. Longi R, Valsasina R, Butte C, Paccenelli S, Riva E, Giovannini M 1985 Cranial computerized tomography in dihydropteridine reductase deficiency. J Inherited Metab Dis 8:109-112

20. Coskun T, Besim A, Ozalp I, Muzaffer E 1990 Intracranial calcification in dihydropteridine reductase deficiency. Turkish J Pediatr 32:259-264

21. Dhondt JL 1991 Strategy for the screening of tetrahydrobiopterin deficiency among hyperphenylalaninemic patients: 15 years' experience. J Inherited Metab Dis 14:117-127

22. Cotton RGH, Jennings I, Bracco G, Ponzone A, Guardamagna O 1986 Tetrahydrobiopterin non-responsiveness in dihydropteridine reductase defi- ciency is associated with the presence of mutant protein. $J$ Inherited Metab Dis 9:239-243

23. Ponzone A, Guardamagna O, Ferraris S, Bracco G, Cotton RGH 1987 Screening for malignant phenylketonuria. Lancet 2:512-513

24. Endres W, Ibel H, Kierat L, Blau N, Curtius HCh 1987 Tetrahydrobiopterin and "non-responsive" dihydropteridine reductase deficiency. Lancet 2:223

25. Ponzone A, Guardamagna O, Ferraris S, Bracco, G, Niederwieser A, Cotton RGH 1988 Two mutations of dihydropteridine reductase deficiency. Arch Dis Child 63:154-157

26. Irons M, Levy H, O'Flynn ME, Stack CV, Langlis PJ, Butler IJ, Milstien S, Kaufman S 1987 Folinic acid therapy in treatment of dihydropteridine reductase deficiency. J Pediatr 110:61-67

27. Woody RC, Brewster MA, Glasier C 1989 Progressive intracranial calcification in dihydropteridine reductase deficiency prior to folinic acid therapy. Neurology 39:673-675

28. Kaufman S 1991 Some metabolic relationships between biopterin and folate: implications for the "methyl trap hypothesis." Neurochem Res 16.1931 1936

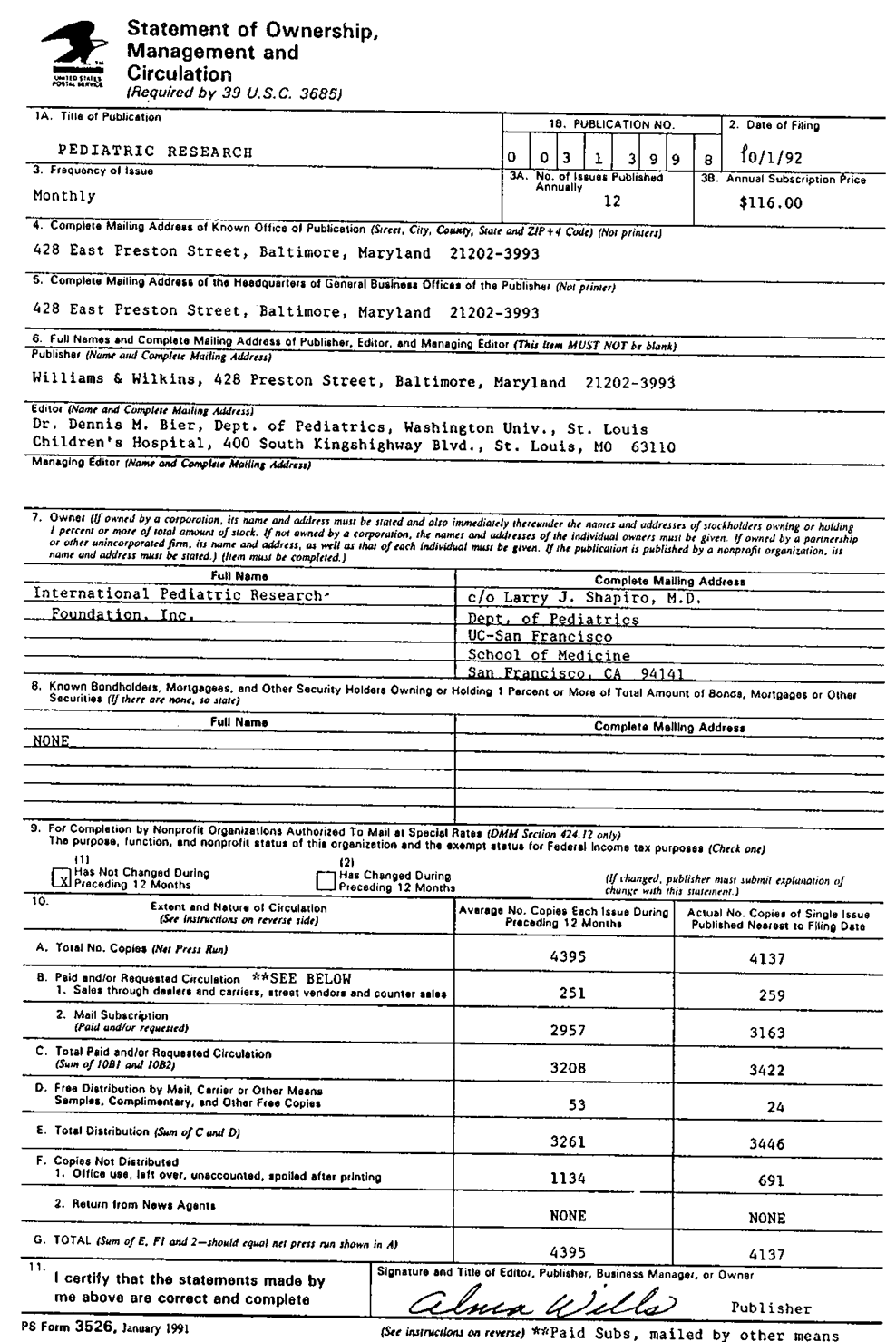

